\title{
A survey of cannabis (marijuana) use and self-reported benefit in men with chronic prostatitis/chronic pelvic pain syndrome
}

\author{
Dean A. Tripp, PhD; ${ }^{*}$ J. Curtis Nickel, MD, FRCSC; ${ }^{\dagger}$ Laura Katz, PhD; $;$ Adrijana Krsmanovic, MSc,; \\ Mark A. Ware, MD, MRCP(UK), MSc, Darcy Santor, PhD
}

*Departments of Psychology, Anesthesiology and Urology, Queen's University, Kingston, ON; 'Department of Urology, Queen's University, Kingston, ON; \$Psychology, Queen's University, Kingston, ON; ${ }^{ \pm}$Alan Edwards Pain Management Unit, McGill University Health Centre, Montreal, QC; *School of Psychology, University of Ottawa, Ottawa, ON

Cite as: Can Urol Assoc J 2014;8(11-12):e901-5. http://dx.doi.org/10.5489/cuaj.2268 Published online December 15, 2014.

\section{Abstract}

Introduction: Chronic prostatitis/chronic pelvic pain syndrome (CP/CPPS) is a chronic pelvic pain condition largely refractory to treatment. Cannabis (marijuana) use has been reported for a wide variety of chronic pain conditions, but no study has examined prevalence of cannabis use, symptom benefit or side effects, or frequency in CP/CPPS.

Methods: Participants were recruited from an outpatient CP/ CPPS urology clinic $(n=98)$ and online through the Prostatitis Foundation website $(n=244)$. Participants completed questionnaires (demographics, CP/CPPS, depression, cannabis).

Results: The clinic sample included Canadian patients and the online sample included primarily American patients. Due to differences, groups were examined separately. Almost 50\% of respondents reported using cannabis (clinic $n=49$; online $n=89$ ). Of the cannabis users, $36.8 \%$ of clinic and $75 \%$ of online respondents reported that it improved their symptoms. Most of the respondents (from the clinic and online groups) reported that cannabis improved their mood, pain, muscle spasms, and sleep. However, they did not note any improvements for weakness, fatigue, numbness, ambulation, and urination. Overall, the effectiveness of cannabis for CP/ CPPS was "somewhat/very effective" (57\% clinic; 63\% online). There were no differences between side effects or choice of consumption and most reported using cannabis rarely.

Conclusions: These are the first estimates in men suffering from CP/ CPPS and suggest that while cannabis use is prevalent, its medical use and benefit are unknown. This is an understudied area and the benefit or hazard for cannabis use awaits further study.

\section{Introduction}

Chronic prostatitis/chronic pelvic pain syndrome (CP/CPPS) is characterized by pain in the perineum, pelvic, and suprapubic areas or the external genitalia with variable degrees of voiding or ejaculatory disturbances. ${ }^{1,2}$ The prevalence is about
7.1\% (range: $2.2 \%-16 \%$ ), with a $6.7 \%$ median. ${ }^{3}$ CP/CPPS symptoms do not routinely remit, with $66 \%$ of communitybased samples experiencing symptoms 1 year later, ${ }^{4}$ and patients showing no changes in pain, disability, or catastrophizing over 2 years later. ${ }^{5} \mathrm{CP} / \mathrm{CPPS}$ etiology is unclear and medical treatments are largely ineffective. ${ }^{6}$ Medications (antimicrobials, alpha-blockers, anti-inflammatories), as well as phytotherapy, biofeedback, thermal therapies, and pelvic floor training have been examined ${ }^{7}$ and may provide mild benefit, ${ }^{6}$ but most men continue to experience chronic pain.

Physicians may use opioids to manage CP/CPPS pain, but their efficacy is limited and physicians fear tolerance, misuse, and side effects, such as nausea/vomiting or sedation. ${ }^{8}$ Chronic pain patients are turning to alternate forms of symptom relief, yet no research on this is available for CP/CPPS. Cannabis sativa has been used for pain and symptom relief for thousands of years. In Canada and several American states, patients use medical cannabis for severe intractable illnesses. As an addition to opioid treatment for chronic pain, vaporized cannabis results in pain reduction without altering plasma opioid levels. ${ }^{9}$ Moreover, $71 \%$ of the available randomized controlled studies concluded that cannabinoids were associated with pain relief, with low adverse effects, and good tolerance. ${ }^{10}$ Cannabis may be used in conjunction with or substitute for prescription opiates resulting in reduced opiate use. ${ }^{11}$ Wide ranging types, quantities, and frequency of cannabis use for pain relief have been reported, with chronic non-cancer pain patients reporting previous use $(15 \%)$ or current use $(10 \%) .^{12}$

We examined cannabis prevalence among men experiencing CP/CPPS-like symptoms from a tertiary care urology department and from an online group. Although previous work has not examined cannabis use in CP/CPPS, it was expected that use would echo previous pain studies. ${ }^{12}$ We also solicited patient self-reports on the side effects or potential benefits, frequency, and indication of future cannabis use. 
Tripp et al.

\section{Methods}

\section{Participants/procedure}

Identical online and outpatient surveys were administered to an online community-based sample and a tertiary care outpatient CP/CPPS clinic sample. All participants remained anonymous and received no financial compensation. Clinic patients were approached after their appointments and briefed about this Research Ethics Board-approved study. Interested participants then provided written consent and received a package (letter of information, debriefing form, questionnaires, postage-paid return envelope) to complete and mail back. The online sample was recruited through the Prostatitis Foundation. ${ }^{13}$ Participants were a self-selected "availability" sample from site visitors. All participants were required to read and write in English. All questionnaires were in English.

\section{Measures}

\section{Demographics}

Participants completed questions on demographics (age, CP/ CPPS diagnosis, health problems, tobacco use, medication use).

\section{Medical symptoms}

The National Institutes of Health Chronic Prostatitis Symptom Index (NIH-CPSI) ${ }^{14}$ assessed prostatitis-like symptoms and their impact on daily life (pain, urinary symptoms, quality of life) providing a score range from 0 to 43 . The selfadministered NIH-CPSI provides a valid, psychometrically robust outcome measure. ${ }^{14}$ Confirmation of CP/CPPS cases was based on NIH-CPSI pain/discomfort in perineum and/or with ejaculation and $\mathrm{NIH}-\mathrm{CPSI}$ total pain score of $\geq 4(0-21)$, used in the community ${ }^{15-17}$ and in the general population ${ }^{18}$ studies.

\section{Depression}

The Patient Health Questionnaire 9 (PHQ-9) ${ }^{19}$ is a reliable and valid self-report measure using 9 items to assess depressive symptoms. An item sum was used for the indexation of depression.

\section{Experience with cannabis}

We used a 21-question descriptive survey on experience with cannabis; questions were binary (yes/no), multiple choice, and rating scales. Questions included whether participants had ever used cannabis, the purpose of use, relief of pain/ effects with use, potential side effects, usage frequency, and usage method. ${ }^{12}$ Participants rated personal experience with different modes of delivery using Likert scale-style responses.

\section{Data analysis}

Scores were excluded if $>15 \%$ of the items were missing on measures. Participants who provided $\geq 85 \%$ of items on a particular measure had the missing items imputed using means replacement procedures. ${ }^{20}$ As a check on generalizability, primary comparisons between the online and clinic data were computed for age and domains of $\mathrm{NIH}-\mathrm{CPSI}$ (quality of life pain, urinary), and the PHQ-9. If outcomes differed significantly, online and clinical samples would be examined separately. Due to the exploratory nature, unadjusted t-tests and descriptive analyses (chi-square) were used to evaluate differences between cannabis users and non-users.

\section{Results}

The total sample of participants was comprised of an online self-reported CP/CPPS sample $(n=376)$ and a tertiary care outpatient CP/CPPS clinic sample $(n=100)$. Two participants were excluded from the clinic sample and $35 \%(n=132)$ of the online sample was excluded due to incomplete data. In the end, we had 244 online and 98 clinic participants. Missing data pattern for the online group was random.

The online group was on average 10 years younger than the clinic group $(p<0.001)$, with an average age of 44.57 (standard deviation 13.96) for the full sample (Table 1). The clinic sample was Canadian and the online sample was primarily American.

The online group reported more depressive symptoms, pain, poorer quality of life, and worse symptom scores (Table $2)$. For the remaining analyses, samples were examined across groups. Most of clinic (63.3\%) and online (79.1\%) participants reported a score of 4 or above on prostatitis cut scores $\left(\chi^{2}=9.24, p=0.013\right)$. While the clinic and online groups did not differ in terms tobacco use $\left(\chi^{2}=1.17\right.$, $p=0.340)$, the online group $(63.1 \%)$ consumed more medication for pain, mood, sleep, or spasms than the clinic group $(47.4 \%)\left(\chi^{2}=7.00, p=0.01\right)$. There were no group differences when asked if they had ever used cannabis $\left(\chi^{2}=0.87\right.$, $p=0.390$ ) (yes $50 \%$ clinic, $44.3 \%$ online). Examining only those previously using, $36.8 \%$ clinic and $75 \%$ of the online groups reported that cannabis improved their symptoms $\left(\chi^{2}=7.63, p=0.006\right)$.

Participants were questioned whether cannabis use made their symptoms "worse/no better" to "slightly/much better" (Table 3). The large majority of online and half of clinic participants reported that cannabis improved their mood by a 


\begin{tabular}{lcc}
\hline Table 1. Sample demographics & & \\
\hline & Online (n= 244) & Clinic (n = 98) \\
\hline Age (years \pm SD) & $42.04(13.33)$ & $51.56(12.21)$ \\
\cline { 2 - 3 } Continent N (\% of column total) & & \\
North America & $162(66 \%)$ & \\
$\quad$ Canada & $12(7 \%)$ & $98(100 \%)$ \\
$\quad$ United States & $150(93 \%)$ & \\
South America & $6(2 \%)$ & \\
Europe & $54(22 \%)$ & \\
Australia & $4(2 \%)$ & \\
Asia & $15(6 \%)$ & \\
Africa (Egypt) & $3(1 \%)$ & \\
\hline SD: standard deviation. &
\end{tabular}

"slightly/much better" degree (Fisher's exact test $p=0.026$ ). Across both groups, cannabis' effects made pain "slightly/ much better" $\left(\chi^{2}=2.48, p=0.619\right)$, as with muscle spasms $\left(\chi^{2}=0.51, p=0.474\right)$, sleep $\left(\chi^{2}=0.54, p=0.461\right)$, and a borderline majority for nausea $\left(\chi^{2}=0.51, p=0.474\right)$. Also a minority of participants reported "slightly/much better" improvement in weakness $\left(\chi^{2}=3.11, p=0.078\right)$, fatigue $\left(\chi^{2}=3.40, p=0.065\right)$, numbness $\left(\chi^{2}=1.16, p=0.281\right)$, ambulating $\left(\chi^{2}=0.64, p=0.423\right)$, and urination (Fisher's exact test $p=0.432)$. When asked on overall effectiveness of cannabis for CP/CPPS, most participants (57\% clinic, $63 \%$ online) reported cannabis as "somewhat/very effective" $\left(\chi^{2}=7.89, p=0.051\right)$.

There was an even distribution of side effects reported by the groups, with most suggesting "none' to "mild" side effects from cannabis use $(70.3 \%$ clinic, $70.8 \%$ online $)\left(\chi^{2}=0.05\right.$, $p=0.972$ ) (Table 4). Also, if offered a choice, participants reported similar preferences for cannabis method across groups $\left(\chi^{2}=1.99, p=0.370\right)$, but smoking was a leading choice. There were no differences when asked about the preferred form of cannabis they had used $\left(\chi^{2}=2.59, p=0.274\right)$, although most participants listed herbal option (buds, sinsemilla, hydrophonic). In current cannabis users, frequency did not differ between groups $\left(\chi^{2}=0.27, p=0.88\right)$, with most respondents using "rarely" ( $73.3 \%$ clinic, $77.3 \%$ online).

\section{Discussion}

This is the first study to document the initial prevalence and patterns of cannabis use in men suffering from CP/CPPS from an outpatient urology clinic and online. Almost 50\% of participants used cannabis and almost $3 / 4$ reported using it for symptom relief. These figures are bigger than those in other studies. ${ }^{12}$ The samples were treated as separate during analyses because the online group was younger, reported greater depression, pain, and diminished quality of life. Interestingly, while examining only those having used cannabis, fewer clinic respondents reported benefit compared to the online group. This almost doubling of the reported
Table 2. Psychological and NIH-CPSI differences between clinic and online participants

\begin{tabular}{lccccc}
\hline & & N & Mean & SD & $p$ value \\
\hline Depressive & Clinic & 92 & 7.15 & 6.66 & $<0.001$ \\
symptoms & Online & 231 & 10.23 & 6.88 & \\
NIH-CPSI pain & Clinic & 98 & 9.04 & 5.79 & $<0.001$ \\
domain & Online & 244 & 11.54 & 4.16 & \\
NIH-CPSI urinary & Clinic & 98 & 4.3061 & 3.43544 & 0.388 \\
domain & Online & 244 & 4.6475 & 2.92547 & \\
NIH-CPSI QoL & Clinic & 98 & 5.8061 & 3.53672 & 0.025 \\
domain & Online & 244 & 6.6680 & 2.04689 & \\
& Clinic & 98 & 19.1531 & 11.13122 & 0.003 \\
NIH-CPSI total & Online & 244 & 22.8566 & 7.23104 & \\
\hline
\end{tabular}

NIH-CPSI: National Institutes of Health Chronic Prostatitis Symptom Inventory; SD: standard deviation; QoL: quality of life.

benefit by the online group may be related to symptom/disease severity differences in this study. The present data cannot describe factors underlying differences in benefit across groups, but this study is consistent with the suggestion that chronic pain is associated with lifetime marijuana use. ${ }^{21}$

Physicians should be aware and question patients on cannabis use. Despite a lack of information on the mechanisms of glycinergic cannabinoids for pain, cannabidiol, a major nonpsychoactive component of cannabis, suppressed chronic inflammatory pain in mice. ${ }^{22}$ Furthermore, the use of cannabis was not associated with analgesic tolerance in rats. ${ }^{22}$ It appears that cannabinoids' anti-inflammatory action

Table 3. Cannabis illness-symptom effects across clinic and online participants

\begin{tabular}{|c|c|c|c|}
\hline & & $\begin{array}{l}\text { "Worse/No } \\
\text { Better" (n) }\end{array}$ & $\begin{array}{l}\text { "Slightly/Much } \\
\text { Better" (n) }\end{array}$ \\
\hline \multirow{2}{*}{ Mood } & Clinic & $50.0 \%(12)$ & $50.0 \%(12)$ \\
\hline & Online & $15.8(3)$ & $84.2 \%(16)$ \\
\hline \multirow{2}{*}{ Pain } & Clinic & $33.3 \%(8)$ & $66.7 \%(16)$ \\
\hline & Online & $26.3 \%(5)$ & $73.7 \%(14)$ \\
\hline \multirow{2}{*}{ Muscle Spasms } & Clinic & $47.8 \%(11)$ & $52.2 \%(12)$ \\
\hline & Online & $36.8 \%(7)$ & $63.2 \%(12)$ \\
\hline \multirow{2}{*}{ Sleep } & Clinic & $25.0 \%(6)$ & $75.0 \%(18)$ \\
\hline & Online & $15.8 \%(5)$ & $84.2 \%(16)$ \\
\hline \multirow{2}{*}{ Nausea } & Clinic & $58.3 \%(14)$ & $41.7 \%(10)$ \\
\hline & Online & $47.4 \%(9)$ & $52.6 \%(10)$ \\
\hline \multirow{2}{*}{ Weakness } & Clinic & $82.6 \%(19)$ & $17.4 \%(5)$ \\
\hline & Online & $57.9 \%(11)$ & $42.1 \%(8)$ \\
\hline \multirow{2}{*}{ Fatigue } & Clinic & $79.2 \%(19)$ & $20.8 \%(5)$ \\
\hline & Online & $52.6 \%(10)$ & $47.4 \%(9)$ \\
\hline \multirow{2}{*}{ Numbness } & Clinic & $78.3 \%(18)$ & $21.7 \%(5)$ \\
\hline & Online & $63.2 \%(12)$ & $36.8 \%(7)$ \\
\hline \multirow{2}{*}{ Ambulating } & Clinic & $79.2 \%(19)$ & $20.8 \%(5)$ \\
\hline & Online & $68.4 \%(13)$ & $31.6 \%(6)$ \\
\hline \multirow{2}{*}{$\begin{array}{l}\text { Problems with } \\
\text { bladder/urination }\end{array}$} & Clinic & $87.5 \%(21)$ & $12.5 \%(3)$ \\
\hline & Online & $73.7 \%(14)$ & $26.3 \%(5)$ \\
\hline
\end{tabular}

Note. Numbers in BOLD represent the majority of respondents in the category row. 
Tripp et al.

\begin{tabular}{|c|c|c|c|c|}
\hline Cannabis side effects & & None (n) & Mild (n) & Moderate-severe (n) \\
\hline & Clinic & $37.0 \%(10)$ & $33.3 \%(9)$ & $29.6 \%(8)$ \\
\hline & Online & $39.3 \%(35)$ & $31.5 \%(28)$ & $29.2 \%(26)$ \\
\hline \multirow[t]{3}{*}{$\begin{array}{l}\text { Preferred cannabis method for } \\
\text { use if offered the choice }\end{array}$} & & Smoked & $\begin{array}{l}\text { Sublingual spray/ } \\
\text { vaporizer/inhaler }\end{array}$ & Rectal suppository/skin patch \\
\hline & Clinic & $47.2 \%(17)$ & $41.7 \%(15)$ & $11.1 \%(4)$ \\
\hline & Online & $61.1 \%(44)$ & $29.2 \%(21)$ & $9.7(7)$ \\
\hline \multirow[t]{3}{*}{ Cannabis form used } & & Hashish & $\begin{array}{c}\text { Herbal } \\
\text { (Leaf and stem) }\end{array}$ & $\begin{array}{c}\text { Herbal } \\
\text { (Buds, sinsemilla, hydrophonic) }\end{array}$ \\
\hline & Clinic & $26.5 \%(9)$ & $17.6 \%(6)$ & $55.9 \%(19)$ \\
\hline & Online & $16.2 \%(12)$ & $29.7 \%(22)$ & $54.1 \%(40)$ \\
\hline
\end{tabular}

stimulates cannabinoid receptors. ${ }^{23}$ However, contrasting results about cannabis side effects discouraged the authors for suggesting its chronic use for pain relief due to associated cognitive deficits and gastrointestinal toxicity. ${ }^{23}$

The online group reported greater distress and NIH-CPSI symptoms, but both groups showed trends where most reported improved symptoms like mood, pain, muscle spasms and sleep. However, no improvements were in weakness, fatigue, numbness, or ambulation. Improved symptoms for some patients might reflect the shared effects that pain/muscle spasm can have in regard to improving sleep and ultimately mood. Current research shows that unresolved chronic pain, continuing disease, obesity, and sleeping problems predict the persistence of pain, while issues like mood are weakly associated. ${ }^{24}$ Of other note, cannabis use was not helpful for urinary symptoms, which can be very bothersome in patients with CP/CPPS.

This survey showed that the side effects of cannabis appear minimal, with most patients reporting "none' to "mild" side effects. More detailed information on the amount of cannabis use, the types used (medical vs. other) would be important to provide a more detailed pattern of examining benefits. If offered a choice on how to use cannabis, participants reported smoking as the preferred methods - this is similar with other studies. ${ }^{25}$ There were no differences by groups the herbal form was endorsed by most respondents. In regard to current frequency of use, most participants reported using cannabis "rarely;" further study into usage patterns may shed some light on whether participants use cannabis primarily to manage pain flares or muscle spasms, or to aid with sleep. If usage is associated with intermittent pains, as flares, then that may reflect the rarity of reported use.

Our study has its limitations. This initial survey cannot qualify the benefits/risks of cannabis use in CP/CPPS, and simply suggests rates for further comparison. Sample size was an issue in some analyses because finer detail in questions, such as symptom benefit, had to be collapsed into 2 categories ("worse/no change" and "slightly/much better") from original categories ("much worse," "slighty worse," "no change," "slightly better," "much better"). Larger samples are necessary to gather more accurate patterns of use and benefit.

Although our samples were not randomized or stratified, they represent tertiary care outpatient males diagnosed with $\mathrm{CP} / \mathrm{CPPS}$, as well as community-based men with CP/CPPSlike symptoms. More online participants reported a prostatitis cut score. Perhaps the clinic men experienced reduced symptoms under the care of a specialist, but there was no opportunity to verify this in our study. Future research should also collect healthcare utilization and previous treatments prior to the onset of cannabis use. This data would allow contrasts and provide insight into medical comorbidities prior to cannabis use. It would also be interesting to examine the associations between psychological pre-cannabis use pain-associated comorbidities, like catastrophizing, and patterns of use.

\section{Conclusion}

This is the first study to examine and report on cannabis usage and benefit in participants with CP/CPPS from a tertiary care and community "availability" sample. The current data suggest that almost $50 \%$ of men with CP/CPPS-like symptoms have used cannabis in their lifetimes and that a minority of clinic patients versus most online participants reported cannabis benefit. Future research should examine larger representative samples to further document usage patterns, fuller CP/CPPS symptom benefit, and associated factors with usage in predictive models. The ultimate study would be a randomized controlled trial prospectively evaluating the efficacy and safety of cannabis compared to either placebo or an active comparator.

Competing interests: Authors declare no competing financial or personal interests.

This paper has been peer-reviewed. 


\section{References}

1. Krieger JN, Egan KJ, Ross SO, et al. Chronic pelvic pains represent the most prominent urogenital symptoms of 'chronic prostatitis'. Urology 1996;48:715-21. http://dx.doi.org/10.1016/S00904295(96)00421-9

2. Krieger JN, Nyberg L, Nickel JC. NIH Consensus definition and classification of prostatitis. JAMA 1999;282:236-7. http://dx.doi.org/10.1001/jama.282.3.236

3. Nickel IC, Shoskes DA, Wagenlehner FME. Management of chronic prostatitis/chronic pelvic pain syndrome (CP/CPPS): The studies, the evidence and the impact. World J Urol 2013;31:747-53. http://dx.doi. org/10.1007/s00345-013-1062-y

4. Nickel IC, Downey JA, Nickel KR, et al. Prostatitis-like symptoms: One year later. BJU Int 2002;90:678-81. http://dx.doi.org/10.1046/i.1464-410X.2002.03007.x

5. Tripp DA, Nickel JC, Shoskes D, et al. A 2-year follow-up of quality of life, pain, and psychosocial factors in patients with chronic prostatitis/chronic pelvic pain syndrome and their spouses. World I Urol 2013;31:733-9. http://dx.doi.org/10.1007/s00345-013-1067-6

6. Anothaisintawee T, Attia J, Nickel JC, et al. Management of chronic prostatitis/chronic pelvic pain syndrome: A systematic review and network meta-analysis. JAMA 2011;305:78-86. http://dx.doi. org/10.1001/jama.2010.1913

7. Schaeffer AJ. Clinical practice. Chronic prostatitis and the chronic pelvic pain syndrome. N Eng J Med 2006;355:1690-8.

8. Nickel JC. Opioids for chronic prostatitis and interstitial cystitis: Lessons learned from the 11th World Congress on Pain. Urology 2006;68:697-701. http://dx.doi.org/10.1016/i.urology.2006.04.013

9. Abrams DI, Couey P, Shade SB, et al. Cannabinoid-opioid interaction in chronic pain. Clin Pharmacol Ther 2011;90:844-51. http://dx.doi.org/10.1038/clpt.2011.188

10. Aggarwal SK. Cannabinergic pain medicine: A concise clinical primer and survey of randomized-controlled trial results. Clin J Pain 2013;29:162-71. http://dx.doi.org/10.1097/AJP.0b013e31824c5e4c

11. Lucas P. Cannabis as an adjunct or substitute for opiates in the treatment of chronic pain. J Psychoactive Drugs 2012;44:125-33. http://dx.doi.org/10.1080/02791072.2012.684624

12. Ware $M A$, Doyle $C R$, Woods $R$, et al. Cannabis use for chronic non-cancer pain: Results of prospective survey. Pain 2003;102:211-6. http://dx.doi.org/10.1016/s0304-3959(02)00400-1

13. Prostatitis Foundation. http://www.prostatitis.org. Accessed December 3, 2014.

14. Litwin MS, McNaughton-Collins M, Fowler FJ Jr, et al. The National Institutes of Health chronic prostatitis symptom index: Development and validation of a new outcome measure. Chronic Prostatitis Collaborative Research Network. J Urol 1999;162:369-75. http://dx.doi.org/10.1016/S0022-5347(05)68562-X
15. Nickel JC, Downey J, Hunter D, et al. Prevalence of prostatitis-like symptoms in a population based study using the National Institutes of Health chronic prostatitis symptom index. J Urol 2001;165:842-5. http:// dx.doi.org/10.1016/S0022-5347(05)66541-X

16. Tripp DA, Nickel JC, Ross $S$, et al. Prevalence, symptom impact and predictors of chronic prostatitislike symptoms in Canadian males aged 16-19 years. BJU Int 2009;103:1080-4. http://dx.doi. org/10.1111/j.1464-410X.2008.08157.x

17. Tripp DA, Nickel JC, Pikard JL, et al. Chronic Prostatitis-like symptoms in African males aged 16-19 years. Can J Urol 2012;19:6081-7.

18. Hu JC, Link C, McNaughton Collins M, et al. The association of abuse and symptoms suggestive of chronic prostatitis/chronic pelvic pain syndrome: Results from the Boston Area Community Health Survey. J Gen Intern Med 2007;22:1532-7. http://dx.doi.org/10.1007/s11606-007-0341-y

19. Spitzer R, Kroenke K, Williams J. Validation and utility of a self-report version of PRIME-MD. JAMA 1999;282:1737-44. http://dx.doi.org/10.1001/jama.282.18.1737

20. Tabachnick BG, Fidell FS. Using multivariate statistics. 5th ed. Boston, MA: Pearson; 2007.

21. Zvolensky MJ, Cougle JR, Bonn-Miller MO, et al. Chronic pain and marijuuana use among a nationally representative sample of adults. Am J Addict 2011;20:538-42. http://dx.doi.org/10.1111/j.1521$0391.2011 .00176 . x$

22. Xiong W, Cui TX, Cheng KJ, et al. Cannabinoids suppress inflammatory and neuropathic pain by targeting alpha 3 receptors. J Exp Med 2012;209:1121-34. http://dx.doi.org/10.1084/jem.20120242

23. Mulvihill MM, Nomura DK. Therapeutic potential of monoacylglycerol lipase inhibitors. Life Sci 2013;92:492-7. http://dx.doi.org/10.1016/i.ffs.2012.10.025

24. Mundal I, Gråwe RW, Biørngaard JH, et al. Prevalence and long-term predictors of persistent chronic widespread pain in the general population in an 11-year prospective study: The HUNT study. BMC Musculoskelet Disord 2014;15:213. http://dx.doi.org/10.1186/1471-2474-15-213

25. Hazekamp A, Ware MA, Muller-Vahl KR, et al. The medicinal use of cannabis and cannabinoids-An international cross-sectional survey on administration forms. J Psychoactive Drugs 2013;45:199-210. http://dx.doi.org/10.1080/02791072.2013.805976

Correspondence: Dr. Dean A. Tripp, Department of Psychology, Queen's University, Kingston, ON K7L 3N6; dean.tripp@queensu.ca 